\title{
Diagnóstico ecográfico de afecciones hepáticas en caninos
}

\author{
Lockett, M.B. ${ }^{\text {; }}$ Koscinczuk, P. ${ }^{2}$; Rosciani, A.S. ${ }^{3}$; Insfrán, R.M..; Repetto, C.J. ${ }^{1}$ \\ ${ }^{1}$ Servicio de Ecografía del Hospital de Clínicas, ${ }^{2}$ Cátedra de Patología Médica, ${ }^{3}$ Servicio de Histopatología y \\ Citología, Facultad de Ciencias Veterinarias, UNNE, Sargento Cabral 2139, Corrientes (3400), Argentina. \\ Tel/Fax 03783-425753. E.mail: mariellock@vet.unne.edu.ar.
}

\begin{abstract}
Resumen
Lockett, M.B.; Koscinczuk, P.; Rosciani, A.S.; Insfrán, R.M.; Repetto, C.J.: Diagnóstico ecográfico de afecciones hepáticas en caninos. Rev. vet. 20: 2, 92-96, 2009. Actualmente, la ecografía es la modalidad de diagnóstico por imagen de primera elección en las patologías hepáticas, ya que permite observar el parénquima, la vesícula biliar y la vasculatura, así como evaluar la relación del hígado con otros órganos. Además, facilita la realización de toma de muestras tanto para citología como histopatología. Este trabajo describe la casuística de alteraciones ecográficas de hígado en caninos que asistieron al Servicio de Diagnóstico por Imágenes del Hospital de Clínica de la Facultad de Ciencias Veterinarias de la Universidad Nacional del Nordeste (Corrientes, Argentina) durante el período 2007-2009. Del total de pacientes que asistieron a la consulta externa del hospital ( $\mathrm{n}=14.627), 52$ casos fueron remitidos por sospechas de patologías hepáticas con síntomas clínicos asociados a alteraciones bioquímicas. De esos 52 pacientes, el 27\% $(\mathrm{n}=14)$ presentó hallazgos ecográficos sólo en hígado, el resto de los casos observados $(73 \%, \mathrm{n}=38)$ presentaron alteraciones tanto en hígado como en otros órganos. El 63\% ( $n=33)$ evidenció aumento del tamaño del hígado, el 8\% $(n=4)$ disminución y el $29 \%$ $(n=15)$ se mantuvo dentro de los límites normales. Referente a las variaciones de la arquitectura del órgano, en un $79 \%$ de los casos la distribución fue heterogénea y en un $21 \%$ homogénea. Respecto a la ecogenicidad hepática, el $81 \%$ evidenció alteraciones difusas y el 19\% alteraciones focales. Las asociaciones de patologías del hígado con vesícula biliar se observaron en el $13 \%$ de los casos $(\mathrm{n}=7)$ y, si bien se trabajó con ecografía convencional, pudieron detectarse pacientes con trastornos vasculares $(27 \%, \mathrm{n}=14)$. Aunque la ecografía hepática presenta ciertas limitaciones en cuanto a especificidad y sensibilidad, brinda información sustancial que permite considerar enfermedades que de otra manera hubieran pasado desapercibidas.
\end{abstract}

Palabras clave: perro, hígado, hepatopatía, ecografía.

\begin{abstract}
Lockett, M.B.; Koscinczuk, P.; Rosciani, A.S.; Insfrán, R.M.; Repetto, C.J.: Ultrasound diagnosis of hepatic canine alteration. Rev. vet. 20: 2, 92-96, 2009. Nowadays, ultrasonography is the diagnostic imaging procedure of first choice in hepatic pathology because it makes possible to assessment the organ structure, gall bladder, vessels, and liver relationship with other organs. Moreover, it makes possible to obtain samples for cytology and/or histopathology. This work describes the hepatic ultrasonographic alterations in dogs that arrived to the Diagnostic Service of Hospital de Clínicas, Facultad de Ciencias Veterinarias, Universidad Nacional del Nordeste (Corrientes, Argentina) during 2007-2009. From the total of patients that arrived to School of Veterinary Hospital) $(n=14,625), 52$ were referred by a probably liver pathology with signs, symptoms and biochemical alterations. From these 52 patients, $27 \%$ $(n=14)$ had signs of hepatic alteration, while $73 \%(n=38)$ showed alterations in both liver and other organs. Considering observations on liver structure, $63 \%(\mathrm{n}=33)$ showed an increment of size, $8 \%(n=4)$ organ-size reduction, and $29 \%(n=15)$ were on the normal limits. Taking into account the variations of the architecture of the liver, $79 \%$ of the cases had heterogenic distribution, while $21 \%$ were homogeneous; regarding hepatic ecogenicity, $81 \%$ of the cases showed diffuse alterations and 19\% focal abnormalities. Pathological associations of liver and gallbladder were observed in $13 \%$ of the cases $(n=7)$ and, although conventional ultrasonography was used for this study, we detected $27 \%(\mathrm{n}=14)$ cases with vascular alterations. Even though liver ultrasonography presents some limitations regarding specificity and sensibility, it gives substantial information that allows to consider some diseases that may go unnoticed.
\end{abstract}

Key words: dog, liver, hepatopathy, ultrasound. 


\section{INTRODUCCIÓN}

El advenimiento de la ecografía como método rutinario para la exploración de las enfermedades hepáticas ha permitido ampliar el campo de las técnicas exploratorias del hígado. Al ser un método inocuo y de alta fiabilidad, se ha situado en primer lugar entre los diferentes procedimientos diagnósticos, complementando la radiografía y el laboratorio bioquímico ${ }^{1,12,17}$.

Por otra parte, la ecografía intervencionista permite la obtención de biopsias de lesiones hepáticas dirigidas por ultrasonido y facilita la aproximación al diagnóstico etiológico ${ }^{8,10,13,15}$. Para la ejecución e interpretación de las imágenes registradas se requiere una combinación de pericia técnica y experiencia. Algunos diagnósticos se efectúan con rapidez y seguridad, otros requieren la comparación de imágenes en exámenes secuenciales ${ }^{4-6,13}$.

La ultrasonografía brinda información referida a alteraciones estructurales que afectan al hígado. Normalmente el parénquima hepático tiene una ecogenicidad homogénea de nivel medio, comparada con la de corteza renal y bazo $2,4,9,13,15$, siendo posible distinguir con relativa facilidad las estructuras ocupadas con líquido de aquellas con tejido blando y sólido, como así también la visualización de anormalidades en la vesícula biliar, vasos hepáticos y órganos adyacentes $4,9,15,17$.

Las alteraciones difusas que afectan al hígado se pueden manifestar con un aumento de la ecogenicidad (hiperecogenicidad), como en los casos de hepatopatía esteroide y lipidosis, o con una disminución (hipoecogenicidad) observada en la congestión pasiva, linfoma y hepatitis supurativa ${ }^{2,3,16}$. Otra de las ventajas de la ultrasonografía es que permite diferenciar entre ictericia obstructiva y no obstructiva mediante la evaluación de la permeabilidad de los canalículos biliares ${ }^{16}$.

Con respecto al tamaño hepático, la ecografía puede distinguir entre hepatomegalia y masas adyacentes al hígado originadas en órganos vecinos ${ }^{9}$. Puede demostrar variantes anatómicas como el aumento de volumen de un hígado normal. Cuando existe una auténtica hepatomegalia la ecografía permite diferenciar entre obstrucción biliar y congestión hepática; en esta última resaltará la distensión de las venas hepáticas y la cava inferior ${ }^{15}$.

Se ha demostrado que la ecografía presenta limitaciones en cuanto a especificidad y sensibilidad para discernir entre las distintas alteraciones del parénquima hepático. Existe un límite potencial para la realización de un diagnóstico específico basado solamente en las características eco-texturales de la lesión. Por ejemplo, la hiperplasia nodular, una lesión sin importancia relacionada con la edad, puede semejarse a una neoplasia. Alteraciones difusas e infiltrativas como las del linfoma pueden pasar desapercibidas porque no alteran la estructura del órgano y muchas veces se manifiestan solamente por variaciones de ecogenicidad, compatibles con otras patologías que afectan a este órgano ${ }^{2,7}$, ${ }^{13}$. En estos casos la utilidad de la ecografía es la de ser una herramienta que permite llegar a un sector determinado del órgano, guiando y optimizando la toma de muestra para citología o histopatología. ${ }^{2,13,15}$.

El propósito de este trabajo fue presentar la casuística de alteraciones ecográficas observadas en el hígado de pacientes caninos con alteraciones bioquímicas de probable origen hepático, que asistieron a un servicio de diagnóstico por imágenes, durante el período 2007-2009.

\section{MATERIAL Y MÉTODOS}

Del total de caninos que asistieron a consultorios externos del Hospital de Clínicas de la Facultad de Ciencias Veterinarias de la UNNE (Corrientes, Argentina) durante el período comprendido entre febrero de 2007 y noviembre de 2009 ( $n=14.627)$, el 7\% de ellos $(n=995)$ fue derivado al Servicio de Ecografía. De ese total, 52 pacientes fueron involucrados como sospechosos de padecer alteraciones hepáticas, considerando sus historias clínicas, síntomas y pruebas de laboratorio.

Los caninos, de ambos sexos y diferentes razas y edades, se presentaron con un ayuno sólido de 12 horas y dos horas de retención de orina. La preparación del paciente se realizó mediante un rasurado desde caudal de la apófisis xifoidea del esternón, en una zona de 10 cm de ancho.

Para el estudio ecográfico se utilizó un equipo Berger 2010 y transductores convexos, microconvexos y lineales, con frecuencias de 5, 6 y 7,5 mhz. Para homogenizar el contacto de la sonda con la zona rasurada se aplicó un gel de acoplamiento acústico en la piel. Los animales fueron posicionados sobre una mesa en decúbito lateral o dorsal y en algunos casos de pie. La técnica de exploración se inició con un barrido completo del abdomen y prosiguió con la investigación del hígado, para lo cual se utilizaron distintas vías de abordaje: por debajo del hipocondrio, mediante cortes transversales y longitudinales y en algunos casos mediante abordajes intercostales.

Las imágenes observadas fueron clasificadas considerando el tamaño, vascularización, características del parénquima, vías biliares, vesícula y relación del hígado con otros órganos. Cuando se consideraron necesarias, se efectuaron punciones percutáneas con aguja para la obtención de muestras citológicas del hígado, previa verificación del tiempo de coagulación.

\section{RESULTADOS Y DISCUSIÓN}

De los 52 pacientes sospechosos de padecer enfermedades hepáticas, el 27\% $(n=14)$ presentó alteraciones exclusivamente en el hígado y el $73 \%$ restante $(n=$ 38) presentó alteraciones tanto en hígado como en otros órganos. Teniendo en cuenta que estos caninos fueron incluidos por sospecha de enfermedad hepática, el hallazgo de un alto número de compromiso de dos o más órganos señala la utilidad de la ecografía para excluir el diagnóstico de enfermedad hepática única y ampliarlo 
Tabla 1. Cuantificación de los hallazgos ecográficos visualizados en hígados caninos.

\begin{tabular}{llcc}
\hline alteraciones observadas & $\mathrm{n}$ & $\%$ \\
\hline \multirow{2}{*}{ tamaño } & aumentado & 33 & 63 \\
hepático & disminuido & 4 & 8 \\
& conservado & 15 & 29 \\
\hline total & heterogénea & 52 & 100 \\
\hline estructura & homogénea & 11 & 79 \\
hepática & & 21 \\
\hline total & hiperecogénica & 23 & 100 \\
\hline \multirow{3}{*}{ ecogenicidad } & hipoecogénica & 7 & 45 \\
hepática & mixta & 12 & 23 \\
& conservada & 10 & 19 \\
\hline total & & 52 & 100 \\
\hline
\end{tabular}

a otras patologías abdominales, en coincidencia con las aseveraciones de otros autores ${ }^{4}$.

La exploración de otros órganos como bazo, páncreas, riñones, glándulas adrenales y linfonódulos es necesaria para determinar las relaciones anatómicas ${ }^{11}$ y poder distinguir entre hepatomegalia y masas adyacentes al hígado originadas en órganos vecinos, como linfoma, tumores renales y adrenales ${ }^{2,3}$ (Figura 1).

Si bien la ecografía no es el mejor método para determinar el tamaño hepático ${ }^{5-7}$, en la casuística investigada existieron más casos de aumentos que de disminuciones. Cabe destacar que cuando existe una auténtica hepatomegalia, la ecografía permite diferenciar entre un hígado congestivo -caso de insuficiencia cardíaca- (Figura 2), de la hepatomegalia que resulta de la obstrucción biliar ${ }^{15,16}$. La hepatomegalia secundaria a tumores hepáticos, infiltraciones, enfermedades infecciosas localizadas o traumáticas, también pueden ser caracterizadas a partir de la imagen ecográfica ${ }^{2,5,15}$.

En la evaluación del parénquima hepático debe tenerse en cuenta su ecogenicidad, que es la suma de

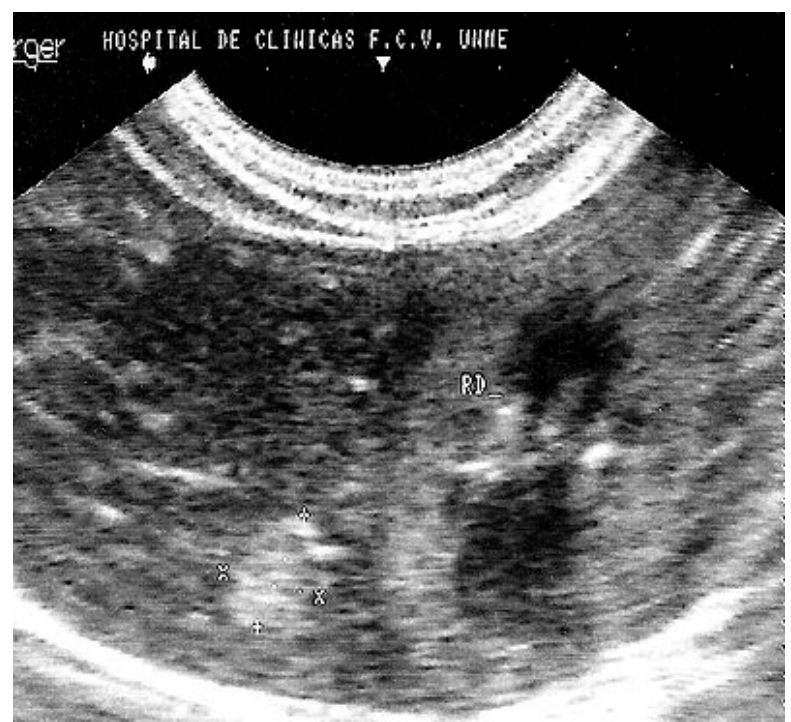

Figura 1. Imagen ecográfica del hígado con una lesión ecogénica focal. Obsérvese también el aumento de la ecogenicidad renal y la relación corticomedular 1:1. la amplitud (tamaño de los ecos) más la distancia que existe entre ellos (densidad ecográfica) ${ }^{15}$. La ecogenicidad del parénquima del hígado, por lo general es igual o mayor que la de la corteza renal y menor que la del bazo. En el presente trabajo hemos observado una mayor cantidad de casos con alteraciones hiperecogénicas (Tabla 1), hallazgos compatibles con sospecha clínica de hepatopatía esteroide, hepatitis crónica y lipidosis ${ }^{4}$. Los patrones mixtos, donde la ecogenicidad está alterada en distintas proporciones, ocurren en algunas inflamaciones difusas, tóxicas o neoplasias, siendo difícil determinar si lo anormal es la mayor o la menor ecogenicidad ${ }^{4}$ . Las alteraciones hipoecogénicas se registraron en menor porcentaje; este patrón ha sido reportado tanto en perros como en gatos con procesos infiltrativos difusos tales como linfomas, leucemia y amiloidosis ${ }^{3,5}$.

Para algunos autores, una de las principales razones para el uso de la ultrasonografía en la evaluación de la enfermedad hepática es diferenciar los procesos locales de los difusos ${ }^{1,16}$. Debe destacarse que en la presente compilación se registró mayor número de pacientes con anormalidades ecogénicas difusas (81\%, Figura 3) que focales (19\%, Figura 4). En las hepatopatías difusas se debe considerar la estructura del parénquima, la cual puede ser homogénea (cuando los ecos se distribuyen regular y uniformemente) o heterogéneas (cuando se distribuyen irregularmente) ${ }^{15}$. La casuística aquí estudiada indica la existencia de mayor número de pacientes que presentaron estructura heterogénea (Figura 5) que homogénea (Tabla 1).

En la identificación de anormalidades del parénquima hepático, tracto biliar y sistema vascular, la ecografía ha llegado a ser una herramienta esencial y, en algunos casos, ha reemplazado a la radiografía como procedimiento screening ${ }^{11}$. Respecto a la relación del hígado con las patologías de la vesícula biliar, estas últimas fueron registradas en 7 de los 52 pacientes con hepatopatías, lo que representa un 13\%. Las alteracio-

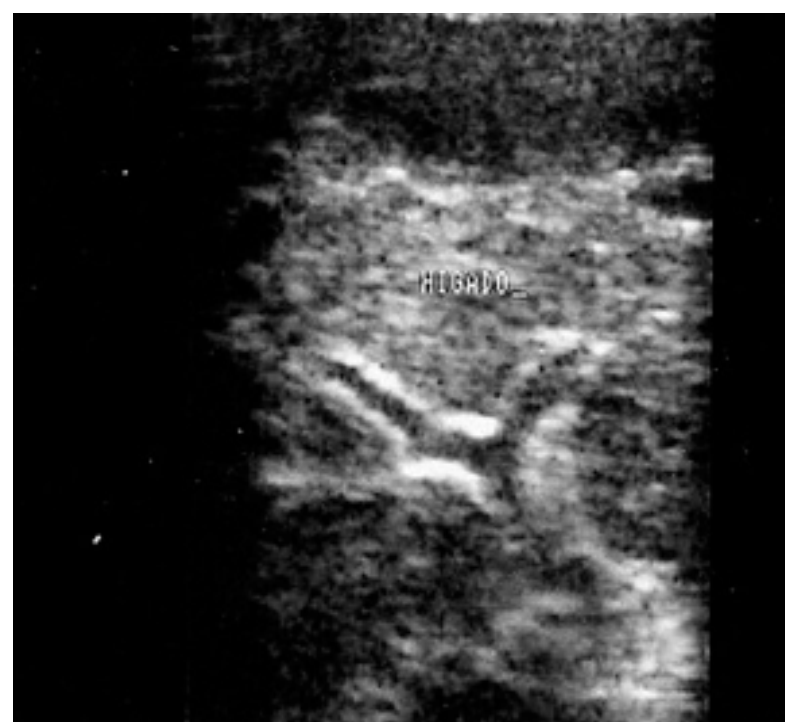

Figura 2. Ultrasonografía hepática donde puede distinguirse dilatación de los vasos portales. 
nes de las vías biliares pueden ser extrahepáticas, donde se observa una marcada distensión de la vesícula biliar acompañada por un cuello tortuoso y dilatado (Figura 6); o intrahepática, como es el caso de la colecistitis ${ }^{13}$.

Si bien con ecografía convencional es posible detectar algunas alteraciones vasculares, es en este punto donde el doppler color (ecodoppler) ha desplazado a la ultrasonografía, aportando imágenes de precisión que permiten evaluar no solo la vasculatura sino también la circulación, teniendo en cuenta la estructura de los vasos, la presión sanguínea y el flujo sanguíneo ${ }^{10,14}$. No obstante, trabajando con ecografía convencional, hemos podido detectar 14 pacientes con trastornos vasculares. La mayoría de los pacientes presentaron congestión pasiva asociada con insuficiencia cardiaca derecha y distensión vascular (Figura 2). Los alcances de la ecografía hepática se ampliarían notablemente

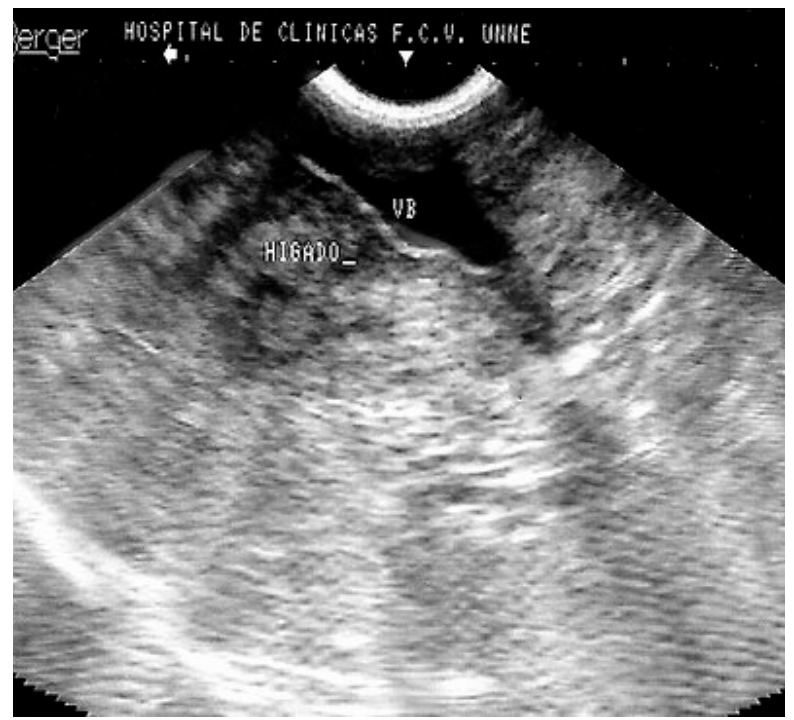

Figura 3. Aumento del volumen hepático. Se observa una textura de distribución difusa e irregular con ecogenicidad aumentada.

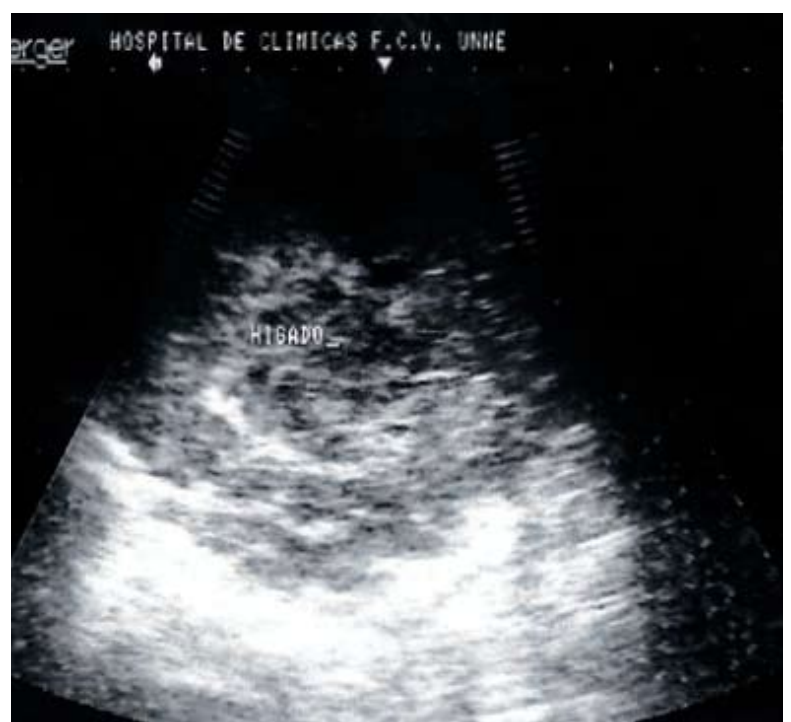

Figura 5. El parénquima hepático se presenta con ecogenicidad mixta y estructura irregular. con el uso de ultrasonografía de contraste o técnicas de ecodoppler ${ }^{10,14}$.

El desarrollo tecnológico de los ecógrafos ha condicionado tanto la precisión diagnóstica de esta técnica como la habilidad clínica para el diagnóstico, siendo una herramienta práctica y segura para los pacientes. Gracias a la ecografía, ahora se pueden considerar enfermedades tales como la colecistitis o colangitis en el diagnóstico diferencial de ictericia, o pueden efectuarse diagnósticos tempranos (algunas veces azarosos) de masas hepáticas cuando examinamos el hígado de un perro con alteraciones bioquímicas y sin sintomatología específica ${ }^{4}$. No obstante, se ha demostrado la existencia de dos grandes limitaciones de la ecografía con respecto a las alteraciones del parénquima hepático: especificidad y sensibilidad y, si bien se puede apreciar una anormalidad, el diagnóstico etiológico final

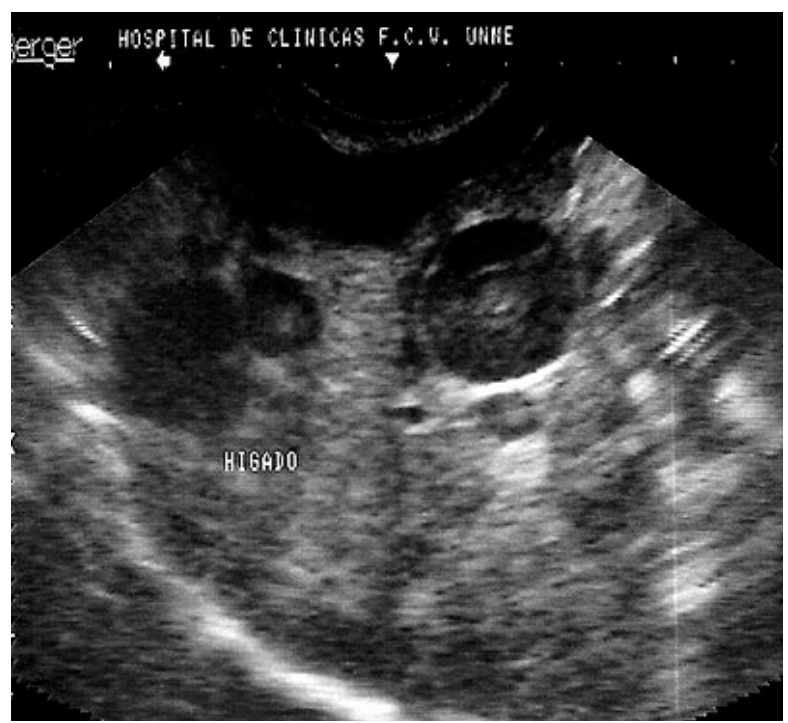

Figura 4. En el hígado se visualizan imágenes nodulares focales de distintos tamaños, algunas hiperecogénicas y otras en forma de diana.

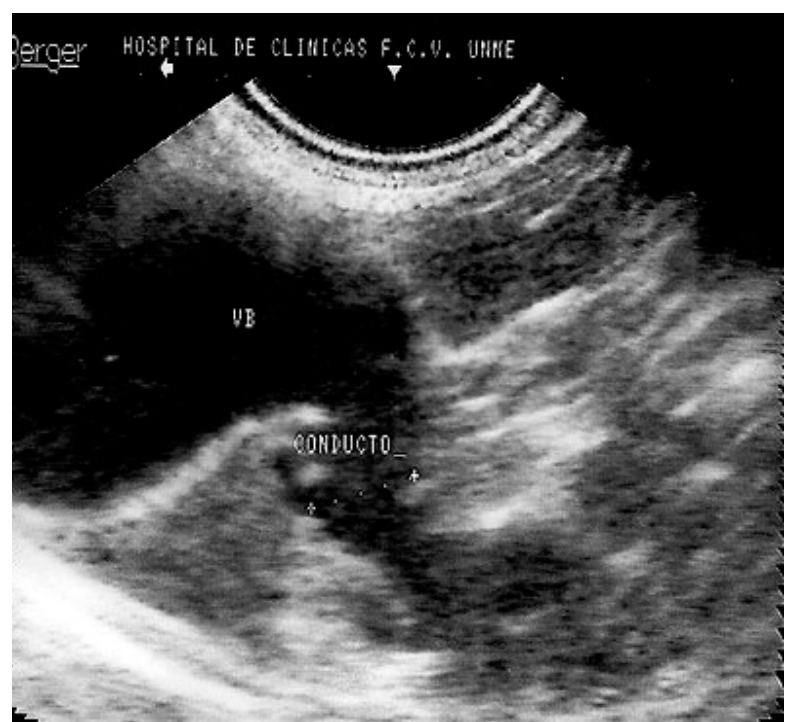

Figura 6. La vesícula biliar se evidencia como una estructura anecogénica de morfología piriforme, con dilatación del conducto. 
con frecuencia depende de los resultados de la biopsia hepática $^{7,9,16}$.

Se postula que para mejorar la eficacia diagnóstica de la ultrasonografía hepática se necesitaría un protocolo estandarizado de investigación. Esto incluiría pautas como selección de la frecuencia óptima del transductor teniendo en cuenta el tamaño del paciente, realización de una observación cuidadosa del parénquima hepático y compararlo con el bazo y la corteza renal, evaluación meticulosa del abdomen, linfonódulos, área pancreática y resto de las estructuras abdominales relacionadas ${ }^{4}$.

En conclusión, se destaca que la ultrasonografía hepática ha llegado a ser una de las herramientas diagnósticas esenciales para identificar anormalidades del parénquima hepático, tracto biliar y sistema vascular.

\section{REFERENCIAS}

1. Cartee RE. 1995. Practical veterinary ultrasound, Williams \& Wilkins, Philadelphia, p. 88-106.

2. Center SA. 1997. Fisiopatología y diagnóstico de los procesos hepatobiliares. En: Tratado de Medicina Interna Veterinaria (Ettinger SJ Ed), $4^{\circ}$ ed., Inter-Médica, Buenos Aires, p. 1525-1589.

3. Farrow C. 2005. Diagnóstico por imagen del perro y el gato, Multimédica Ediciones Veterinarias, Barcelona, p. 627-669.

4. Faverzani S, Chinosi S, Lodi M. 2006. The role of hepatic ultrasonography in small animal medicine: from the identification of the lesions to diagnosis. Vet Res Comm 30: 139-143.

5. Fritsch R. 1996. Ecografía de perros y gatos, Multimédica Ediciones Veterinarias, Zaragoza, p. 98-135.
6. Giraldo CE. 2003. Principios básicos de ultrasonografia veterinaria. Med Vet Zoot (Córdoba, Argentina) 8: 303309.

7. Goddard PJ. 1995. Ecografía veterinaria, Acribia, Zaragoza, p. 43-49.

8. Hardie E, Hahulski-Jaeker G. 2004. Técnicas de biopsia hepática. Waltham Focus 14: 36-42.

9. Khalilov S, Alkan Z, Bumin A, Sen Y, Senel OO, Ince BF. 2008. Evaluation of liver tumors with radiography, ultrasonography and laparoscopy in cats and dogs. Proceedinas 15 th Congress of FAVA, Bangkok (Thailand), p. 29-30.

10. King AM. 2006. Development, advances and applications of diagnostic ultrasound in animals. The Vet $J$ 171: 408 420.

11. Koyama H. 2004. Ultrasonographic evaluation of abdominal organs in veterinary medicine. Int Congr Series 1274: 192-194.

12. Nelson WR, Couto CG. 2005. Medicina interna de animales pequeños, $3^{\circ}$ ed., Inter-Médica, Buenos Aires, p. 517-540.

13. Nyland TG. 2006. Diagnóstico ecográfico en pequeños animales, Multimédica Ediciones Veterinarias, Barcelona, p. $98-135$.

14. Ohlerth S, O'Brien RT. 2007. Contrast ultrasound: general principles and veterinary clinical applications. The Vet $J$ 174: 501-512.

15. Roca Martínez FJ. 1989. Ecografía clínica del abdomen, $2^{\circ}$ ed., Ed. Jims, Barcelona, p. 148-165.

16. Tams TR. 1998. Manual de gastroenterología en animales pequeños, Inter-Médica, Buenos Aires, p. 79-99.

17. Stzatmari V. 2005. Ecografía hepática en perros y gatos. Waltham Focus 15: 2-9. 